\title{
Viable Cell per Femur
}

National Cancer Institute

\section{Source}

National Cancer Institute. Viable Cell per Femur. NCI Thesaurus. Code C73786.

A unit of cell concentration expressed as the number of viable cells per femur. 